\title{
Impact of age on the association between cardiac high-energy phosphate metabolism and cardiac power in women
}

\author{
Maria Nathania, ${ }^{1}$ Kieren G Hollingsworth, ${ }^{1}$ Matthew Bates, ${ }^{2}$ Christopher Eggett, \\ Michael I Trenell, ${ }^{1}$ Lazar Velicki, ${ }^{3}$ Petar M Seferovic, ${ }^{4}$ Guy A MacGowan, ${ }^{5}$ \\ Doug M Turnbull, ${ }^{6,7}$ Djordje G Jakovljevic ${ }^{1,6,8}$
}

For numbered affiliations see end of article.

\section{Correspondence to} Dr Djordje G Jakovljevic Institute of Cellular Medicine, Newcastle Cardiovascular Research Centre, 4th Floor William Leech Building, The Medical School, Newcastle University, Newcastle upon Tyne NE2 4HH, UK; d.jakovljevic@ ncl.ac.uk

Received 26 January 2017 Revised 24 April 2017 Accepted 25 April 2017 Published Online First 12 June 2017

\section{Linked}

- http://dx.doi.org/10.1136/ heartjnl-2017-311729

\section{CrossMark}

\section{To cite: Nathania $\mathrm{M}$} Hollingsworth $\mathrm{KG}$, Bates $M$, et al. Heart 2018;104:111-118.

\section{ABSTRACT}

Objective Diminished cardiac high-energy phosphate metabolism (phosphocreatine-to-ATP (PCr:ATP) ratio) and cardiac power with age may play an important roles in development of cardiac dysfunction and heart failure. The study defines the impact of age on PCr:ATP ratio and cardiac power and their relationship.

Methods Thirty-five healthy women (young $\leq 50$ years, $n=20$; and old $\geq 60$ years, $n=15$ ) underwent cardiac MRI with ${ }^{31} \mathrm{P}$ spectroscopy to assess PCr:ATP ratio and performed maximal graded cardiopulmonary exercise testing with simultaneous gas-exchange and central haemodynamic measurements. Peak cardiac power output, as the best measure of pumping capability and performance of the heart, was calculated as the product of peak exercise cardiac output and mean arterial blood pressure.

Results PCr:ATP ratio was significantly lower in old compared with young age group $(1.92 \pm 0.48$ vs $2.29 \pm 0.55, p=0.03)$, as were peak cardiac power output $(3.35 \pm 0.73$ vs $4.14 \pm 0.81 \mathrm{~W}, p=0.01)$, diastolic function (ie, early-to-late diastolic filling ratio, $1.33 \pm 0.54$ vs $3.07 \pm 1.84, p<0.01)$ and peak exercise oxygen consumption $(1382.9 \pm 255.0$ vs $1940.3 \pm 434.4 \mathrm{~mL} /$ min, $p<0.01)$. Further analysis revealed that PCr:ATP ratio shows a significant positive relationship with early-to-late diastolic filling ratio $(r=0.46, p=0.02)$, peak cardiac power output $(r=0.44, p=0.02)$ and peak oxygen consumption ( $r=0.51, p=0.01)$.

Conclusions High-energy phosphate metabolism and peak power of the heart decline with age. Significant positive relationship between PCr:ATP ratio, early-tolate diastolic filling ratio and peak cardiac power output suggests that cardiac high-energy phosphate metabolism may be an important determinant of cardiac function and performance.

\section{INTRODUCTION}

Heart failure is a clinical syndrome associated with cardiac dysfunction at rest and/or in response to stress. Neubauer proposed that the heart failure is 'an engine out of fuel' and diminished cardiac high-energy phosphate metabolism plays an important role in the mechanisms of heart failure. ${ }^{1}$ The heart has one of the largest metabolic demands in the body and it uses chemical energy stored in phosphoryl bonds of metabolites from its primary substrate (ie, fatty acid, carbohydrate and ketone bodies) to maintain its contractile function. ${ }^{2}$ ATP followed by phosphocreatine (PCr) are the two most important high-energy phosphates in the heart. ${ }^{2}$ Derangements in cardiac metabolism deprive the heart of high-energy phosphates required to maintain its function and performance and may cause mechanical failure of the heart. ${ }^{3}$ Studies have also shown that all components of cardiac metabolism (ie, substrate utilisation, mitochondrial oxidative phospshorylation, high-energy phosphate metabolism and PCr:ATP ratio) are impaired in heart

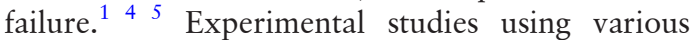
animal models demonstrated the link between cardiac energetics and cardiac function. ${ }^{67}$ Glucose 6-phosphate dehydrogenase (G6PD) is a major key enzyme in NAPDH generation, and G6PD-deficient mice exhibited diminished contractile function and high oxidative stress. ${ }^{6}$ Improvement in cardiac metabolism may lead to better cardiac function, whereas diminished cardiac energy metabolism is a cause of heart failure. ${ }^{6}$

Currently, limited number of clinical studies have investigated the association between cardiac high-energy phosphate metabolism and cardiac function and performance. In particular, no study reported the relationship between PCr:ATP ratio and peak cardiac power output, which is proposed to be the best indicator of overall function and pumping capability of the heart, and the strongest predictor of prognosis in heart failure. ${ }^{8}$ Better understanding of the interaction between metabolism and function of the heart may lead to development of new treatment strategies to improve clinical outcomes. Therefore, the aim of the present study is to first, define the effect of age on cardiac high-energy phosphate metabolism and peak cardiac power output and second, assess the relationship between PCr:ATP ratio and cardiac function and performance. Because of a significant gender difference in age-associated changes in cardiac morphology and function, ${ }^{9}$ we designed this study to address the relationship between cardiac metabolism and performance in women.

\section{METHODS}

\section{Study design}

This was a single-centre, cross-sectional, observational study. 


\section{Participants}

Thirty-five healthy women were recruited as volunteers for the study and grouped according to age (young $\leq 50$ years old, $\mathrm{n}=20$; and old $\geq 60$ years old, $\mathrm{n}=15$ ). Subjects were included in the study only if they (1) had no history of cardiovascular disease, pulmonary diseases and other chronic diseases and (2) had normal glucose tolerance and lipid profile, normal resting blood pressure, normal ECG and body mass index $\leq 30 \mathrm{~kg} / \mathrm{m}^{2}$. Subjects were excluded from the study if they (1) were current or past smokers; (2) were taking any medication known to affect cardiovascular function or (3) were not able to perform maximal graded cardiopulmonary exercise stress test. All participants signed an informed consent according to the Declaration of Helsinki, and the study was approved by the National Health Service North East England-Tyne and Wear South.

\section{Procedures}

All study participants underwent cardiac MRI examination using a 3.0T Philips Intera and a 6-channel cardiac coil (Philips; Best, Netherlands (NL)) with ECG gating. Patients were asked to lie in a supine position and hold their breath while images were obtained in a short axis view. A ViewForum workstation (Philips) was used for cardiac analysis. Epicardial and endocardial borders were manually traced at end-systole and end-diastole on the short axis slices to calculate measures of cardiac function and structure. Contour selection and calculation of left-ventricular mass, systolic and diastolic parameters were performed following previously used protocol. ${ }^{1011}$

Cardiac high-energy phosphate metabolism was assessed using ${ }^{31} \mathrm{P}$ magnetic resonance spectroscopy. Data were collected using a $10 \mathrm{~cm}$ diameter ${ }^{31} \mathrm{P}$ surface coil (Pulseteq, UK) for transmission/ reception of signal. Subjects were placed in a prone position. Shimming was performed using a cardiac triggered, breath-held field map. A slice-selective, cardiac-gated 1-dimensional chemical shift imaging (1D-CSI) sequence was used with a $7 \mathrm{~cm}$ slice selective pulse applied foot head to eliminate contamination from the liver, with spatial presaturation of lateral skeletal muscle. Sixteen coronal phase-encoding steps were used, yielding spectra from $10 \mathrm{~mm}$ slices (repitition time $(\mathrm{TR})=$ heart rate, 192 averages at the centre of $\mathrm{k}$-space with acquisition weighting and $\sim 20 \mathrm{~min}$ acquisition time). Spectral locations were overlaid onto an anatomical image, and the first spectrum arising entirely beyond the chest wall was selected. Quantification of PCr, the $\gamma$ resonance of ATP and 2,3-diphosphoglycerate (DPG) was performed using the AMARES time-domain fit routine in the jMRUI-processing software. Corrections for saturation, flip angle and blood ATP content were made as published previously. ${ }^{12}$

In addition to cardiac MRI, all participants performed maximal graded cardiopulmonary exercise test on an electromagnetically controlled semirecumbent cycle ergometer.
Throughout the exercise test, gas exchange measurement system (Metalyzer 3B, Cortex, Leipzig, Germany) and the bioreactance method (NICOM, Cheetah Medical, Delaware) were used to non-invasively measure gas exchange and central haemodynamics. The bioreactance method accurately measures frequency of relative phase shift of oscillating electronic current across the thorax, and we have recently reported its validity and reliability to assess cardiac output at rest and during exercise. ${ }^{13-16}$ It has greater signal-to-noise ratio by 100 -fold compared with the older bioimpedance method and is less susceptible to disruption from excessive movements, adipose tissue and electrode placement. ${ }^{15}$ Throughout the test, 12-lead electrocardiogram (Custo, CustoMed, GmbH, Ottobrunn, Germany) and blood pressure (Tango, SunTech Medical; Morrisville, North Carolina, USA) were recorded. During the exercise test, participants were asked to cycle at $20 \mathrm{~W}$ for $3 \mathrm{~min}$ as a warm-up period, followed by increase in workload of $10 \mathrm{~W}$ per minute, until volitional exhaustion. Cardiopulmonary exercise test was terminated when (1) subject has reached volitional exhaustion, that is inability to pedal at cadence of 50 revolutions per minute; (2) maximal oxygen consumption was achieved, that is there was no further increase in oxygen utilisation despite increase in exercise intensity (watts); (3) respiratory exchange ratio $>1.15$ or (4) subject voluntarily terminated the test. Cardiac power output, expressed in watts, was calculated as the product of peak exercise cardiac output and mean arterial blood pressure. ${ }^{8}$ Cardiac reserve was defined as the difference between peak and resting cardiac power output. Peak oxygen consumption, as a measure of metabolic response, was defined as the average oxygen uptake during the last minute of exercise.

Physical activity level and number of steps were assessed objectively using a validated portable multisensor array (Sensewear, Bodymedia, Pennsylvania). The monitor was worn for 7 days and was only removed for bathing.

\section{Statistical analysis and sample size}

All statistical analysis was carried out using SPSS V.21.0. Prior to statistical analysis, data were tested for univariate and multivariate outliers using standard Z-distribution cut-offs and Mahalanobis distance tests. Kolmogorov-Smirnov test was used to assess normality of distribution. Differences between age groups were assessed using t-test. Pearson coefficient of correlation was used to assess the relationship between variables. Statistical significance was indicated if $p<0.05$. Data are presented as mean \pm SD unless stated otherwise. A previous study, in which a similar outcome was used, reported a mean difference of 0.24 in PCr:ATP ratio to be significant between younger and older age groups. ${ }^{17}$ To show this difference with at least $70 \%$ power at the $5 \%$ significance, we needed data for a total of 28 participants (ie, $\geq 14$ participants per age group).

Table 1 Participant demographic and clinical measures

\begin{tabular}{lllll}
\hline & All participants & Young $(\leq 50$ years old, $\mathrm{n}=20)$ & Older $(\geq 60$ years old, $\mathrm{n}=15)$ & $\mathrm{p}$ Value \\
\hline Age $($ years $)$ & $55.7 \pm 14.0(40-81)$ & $44.4 \pm 3.2(40-50)$ & $70.9 \pm 5.7(63-81)$ & 0.00 \\
\hline Weight $(\mathrm{kg})$ & $67.7 \pm 11.2(45-93)$ & $69.2 \pm 12.3(53-93)$ & $65.6 \pm 9.6(45-82)$ & 0.35 \\
\hline Height $(\mathrm{cm})$ & $163.0 \pm 5.8(149-175)$ & $166.0 \pm 4.8(155-175)$ & $159.2 \pm 4.7(149-165)$ \\
\hline Body mass index $\left(\mathrm{kg} / \mathrm{m}^{2}\right)$ & $25.5 \pm 3.7(17.9-30.4)$ & $25.2 \pm 4.0(19-30.4)$ & $25.9 \pm 3.4(18-29.8)$ & 0.00 \\
\hline Body surface area $\left(\mathrm{m}^{2}\right)$ & $1.7 \pm 0.1(1.5-2.1)$ & $1.8 \pm 0.1(1.6-2.2)$ & $1.7 \pm 0.1(1.5-1.9)$ & 0.59 \\
\hline Fat body mass $(\mathrm{kg})$ & $23.6 \pm 9.1(5.3-34.1)$ & $22.7 \pm 10.1(5.8-34.1)$ & $24.8 \pm 7.7(5.3-31.2)$ & 0.06 \\
\hline Physical activity $($ steps/day) & $11532 \pm 5422(7694-16348)$ & $11578 \pm 4985(7882-16348)$ & $11651 \pm 5012(7694-15854)$ & 0.51 \\
\hline
\end{tabular}


Table 2 Measures of cardiac structure, function and metabolism

\begin{tabular}{lllll} 
& $\begin{array}{l}\text { All } \\
\text { participants }\end{array}$ & $\begin{array}{l}\text { Young } \\
(\leq 50 \text { years } \\
\text { old, } \mathrm{n}=20)\end{array}$ & $\begin{array}{l}\text { Old } \\
(\geq 60 \text { years } \\
\text { old, } \mathrm{n}=15)\end{array}$ & p Value \\
\hline Left ventricular mass $(\mathrm{g})$ & $85.5 \pm 14.9$ & $90.7 \pm 16.1$ & $78.6 \pm 9.7$ & 0.01 \\
\hline $\begin{array}{l}\text { Eccentricity ratio }(\mathrm{g} / \mathrm{mL}) \\
0.8 \pm 0.1\end{array}$ & $0.8 \pm 0.1$ & $0.8 \pm 0.1$ & 0.16 \\
\hline $\begin{array}{l}\text { Left-ventricular ejection } \\
\text { fraction (\%) }\end{array}$ & $61.7 \pm 7.7$ & $58.7 \pm 7.6$ & $65.8 \pm 5.8$ & 0.01 \\
\hline $\begin{array}{l}\text { PCr:ATP ratio } \\
\text { Longitudinal shortening }\end{array}$ & $2.1 \pm 0.5$ & $2.3 \pm 0.6$ & $1.9 \pm 0.5$ & 0.03 \\
(\%) & $19.4 \pm 5.9$ & $19.4 \pm 3.0$ & $19.5 \pm 4.9$ & 0.92 \\
\hline E:A ratio & $2.3 \pm 1.7$ & $3.1 \pm 1.8$ & $1.3 \pm 0.5$ & 0.00 \\
\hline
\end{tabular}

E;A,early-to-late diastolic filling ratio; PCr:ATP, phosphocreatine-to-ATP.

We have therefore recruited 35 participants (15 younger and 20 older) for the study.

\section{RESULTS}

Data were normally distributed and not outliers were identified in variables of interests. Participants' demographic details are presented in table 1. Anthropometric measures were not significantly different between young and old groups except height. Other measures, such as weight, body mass index, body surface area, fat body mass and lean body mass, were not significantly different between the two groups $(p>0.05)$. The level of physical activity (number of steps per day) was also not significantly different between younger and older women (table 1).

\section{The effect of age on cardiac structure, function and metabolism}

Cardiac MRI revealed that left-ventricular mass and early-to-late diastolic filling ratio were significantly lower in older women compared with younger women $(78.6 \pm 9.7$ vs $90.7 \pm 16.1 \mathrm{~g}$ $\mathrm{p}=0.01$; and $1.3 \pm 0.5$ vs $3.1 \pm 1.8, \mathrm{p}<0.01$, table 2 ). More importantly, older women demonstrated significantly lower PCr:ATP ratio compared with younger women $(1.9 \pm 0.5$ vs $2.3 \pm 0.6$, $\mathrm{p}=0.03$, table 2 , figure $1 \mathrm{~A}$ ).

Resting measures taken prior to cardiopulmonary exercise test revealed that metabolic measures that is, oxygen consumption and respiratory exchange ratio, were not significantly different between young and old groups ( $>>0.05$, table 3$)$. Resting cardiac variables such as stroke volume and cardiac output were significantly lower in the older age group $(70.3 \pm 7.9 \mathrm{vs}$ $87.1 \pm 14.4 \mathrm{~mL} /$ beats, $\mathrm{p}<0.01$ and $4.9 \pm 0.8$ vs $61.1 \pm 1.2 \mathrm{~L} /$ min, $\mathrm{p}<0.01)$. Older women also demonstrated higher systolic blood pressure and systemic vascular resistance $(142.9 \pm 12.5$ vs
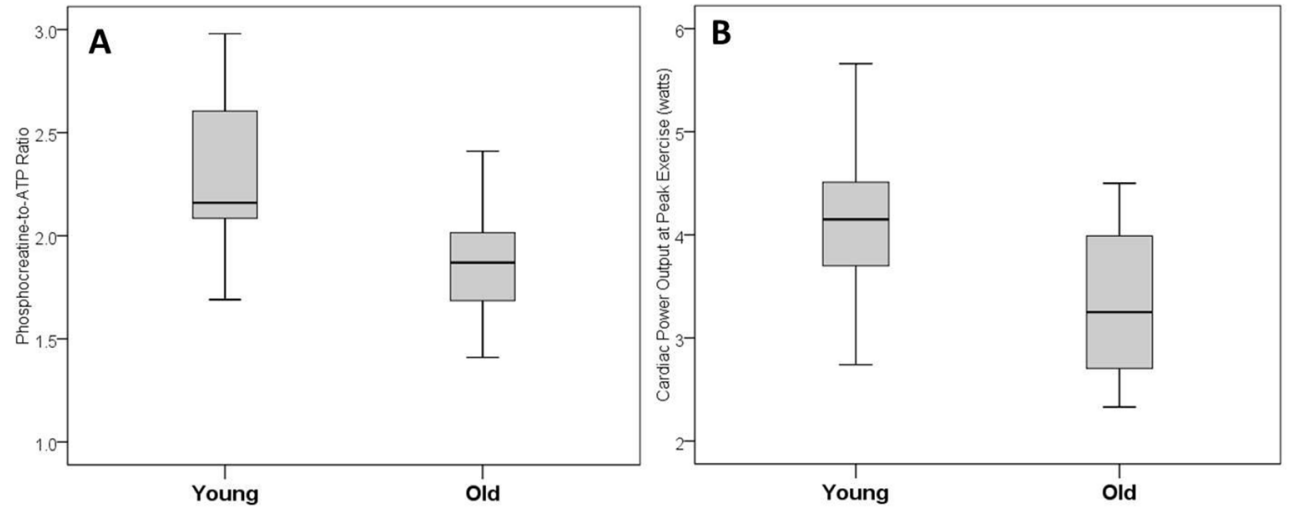

Figure 1 Cardiac high-energy phosphate metabolism—-phosphocreatine-to-ATP ratio (A) and cardiac pumping capability—cardiac power output (B) in young and older women.

Table 3 Resting gas exchange and central haemodynamics measures

\begin{tabular}{|c|c|c|c|c|}
\hline & All participants & Young ( $\leq 50$ years old, $n=20$ ) & Old ( $\geq 60$ years old, $n=15$ ) & p Value \\
\hline \multicolumn{5}{|l|}{ Resting metabolic variables } \\
\hline Oxygen consumption (mL/min) & $244.3 \pm 35.9$ & $250.2 \pm 40.9$ & $236.5 \pm 27.4$ & 0.20 \\
\hline Oxygen consumption (mL/kg/min) & $3.7 \pm 0.7$ & $3.7 \pm 0.9$ & $3.7 \pm 0.5$ & 0.81 \\
\hline Respiratory exchange ratio & $0.9 \pm 0.1$ & $0.9 \pm 0.1$ & $0.9 \pm 0.1$ & 0.90 \\
\hline \multicolumn{5}{|l|}{ Resting haemodynamics } \\
\hline Heart rate (beats/min) & $69.9 \pm 7.6$ & $69.6 \pm 7.6$ & $70.3 \pm 7.9$ & 0.80 \\
\hline Stroke volume (mL/beat) & $80.1 \pm 16.2$ & $87.1 \pm 14.4$ & $70.8 \pm 14.1$ & 0.00 \\
\hline Stroke volume index $\left(\mathrm{mL} /\right.$ beat $\left./ \mathrm{m}^{2}\right)$ & $46.2 \pm 8.3$ & $49.4 \pm 8.0$ & $42.1 \pm 6.8$ & 0.01 \\
\hline Cardiac output (L/min) & $5.6 \pm 1.2$ & $6.1 \pm 1.2$ & $4.9 \pm 0.8$ & 0.00 \\
\hline Cardiac index $\left(\mathrm{L} / \mathrm{min} / \mathrm{m}^{2}\right)$ & $3.2 \pm 0.6$ & $3.4 \pm 0.6$ & $2.9 \pm 0.4$ & 0.00 \\
\hline Systolic blood pressure (mm Hg) & $134.7 \pm 16.6$ & $128.5 \pm 16.8$ & $142.9 \pm 12.5$ & 0.01 \\
\hline Diastolic blood pressure $(\mathrm{mm} \mathrm{Hg})$ & $86.0 \pm 9.9$ & $85.6 \pm 9.9$ & $86.7 \pm 10.1$ & 0.75 \\
\hline Mean arterial blood pressure $(\mathrm{mm} \mathrm{Hg})$ & $102.2 \pm 10.7$ & $99.9 \pm 10.7$ & $105.4 \pm 10.2$ & 0.13 \\
\hline Cardiac power output (W) & $1.3 \pm 0.3$ & $1.4 \pm 0.3$ & $1.2 \pm 0.2$ & 0.06 \\
\hline Cardiac power output index $\left(\mathrm{W} / \mathrm{m}^{2}\right)$ & $0.7 \pm 0.2$ & $0.8 \pm 0.2$ & $0.7 \pm 0.1$ & 0.13 \\
\hline Systemic vascular resistance $($ dyne/(s.cm5 $)$ ) & $1529.0 \pm 359.0$ & $1352.1 \pm 265.6$ & $1764.9 \pm 336$ & 0.00 \\
\hline
\end{tabular}


$128.5 \pm 16.8 \mathrm{~mm} \mathrm{Hg}, \mathrm{p}=0.01$ and $1765 \pm 336 \mathrm{vs} 1352 \pm 266$ dyne $/ \mathrm{s} / \mathrm{cm}^{5}, \mathrm{p}<0.01$; table 3$)$.

At peak exercise, older women exhibited significantly lower oxygen consumption compared with younger women $(21.5 \pm 4.6$ vs $28.6 \pm 8.8 \mathrm{~mL} / \mathrm{kg} / \mathrm{min}, \mathrm{p}<0.01$, table 4$)$. Older women also showed significantly lower peak heart rate $(140 \pm 15$ vs $169 \pm 10$ beats $/ \mathrm{min}, \mathrm{p}<0.01)$, peak cardiac output $(11.9 \pm 2.3$ vs $15.4 \pm 3.0 \mathrm{~L} / \mathrm{min}, \mathrm{p}<0.01)$ and peak cardiac power output $(3.3 \pm 0.7$ vs $4.1 \pm 0.8 \mathrm{~W}, \mathrm{p}=0.01$, table 4 , figure $1 \mathrm{~B})$. Figures 2 and 3 present box-plots (median, first and third quartiles) for the rest and peak exercise variables, that is oxygen consumption, cardiac power output, cardiac index, heart rate, mean arterial blood pressure and systemic vascular resistance.

\section{Relationship between age and cardiac metabolism, function and performance}

Age was significantly associated with decline in PCr:ATP ratio $(r=-0.40, p=0.03)$, early-to-late diastolic filling ratio $(\mathrm{r}=-0.74, \mathrm{p}<0.01)$ and cardiac output $(\mathrm{r}=-0.35, \mathrm{p}=0.04)$. There was a significant positive relationship between age and resting systolic blood pressure $(\mathrm{r}=0.47, \mathrm{p}=0.02)$ and systemic vascular resistance $(r=0.42, p=0.03)$.

At peak exercise, age was significantly associated with lower heart rate $(\mathrm{r}=-0.82, \mathrm{p}<0.01)$, cardiac output $(\mathrm{r}=-0.60$, $\mathrm{p}<0.01)$, cardiac power output $(\mathrm{r}=-0.44, \mathrm{p}<0.01)$ and oxygen consumption $(r=-0.70, p<0.01)$. There was however a positive relationship between age and peak exercise systolic blood pressure $(r=0.38, p=0.04)$ and systemic vascular resistance $(r=0.64$, $\mathrm{p}<0.01)$.

\section{Relationship between cardiac metabolism and cardiac function and performance}

There was no significant relationship between PCr:ATP ratio and measures of cardiac structure and function, except for earlyto-late diastolic filling ratio as a measure of diastolic function $(r=0.46, p=0.02)$. Further analysis revealed a significant relationship between PCr:ATP ratio and measures of cardiac performance, that is peak cardiac power output $(r=0.44, p=0.02$, figure $4 \mathrm{~A})$, peak heart rate $(\mathrm{r}=0.42, \mathrm{p}=03$, figure $4 \mathrm{~B})$, peak oxygen consumption $(r=0.51, p=0.01$, figure $4 C)$, cardiac output $(\mathrm{r}=0.45, \mathrm{p}=0.01)$ and cardiac reserve $(\mathrm{r}=0.42, \mathrm{p}=0.03)$. There was a significant negative relationship between PCr:ATP ratio and systemic vascular resistance $(r=-0.39, p=0.04$, figure 4D). PCr:ATP ratio was significantly correlated with systolic blood pressure at rest $(\mathrm{r}=-0.36, \mathrm{p}=0.04)$, but not at peak exercise $(\mathrm{r}=-0.22, \mathrm{p}=0.09)$.

\section{DISCUSSION}

This is the first study to address the relationship between high-energy phosphate metabolism and maximal performance of the heart assessed in response to cardiopulmonary exercise testing. The study is clinically relevant as it helps better understand the role of high-energy phosphate metabolism in overall function and performance of the heart, further supporting previous suggestion that diminished cardiac metabolism is a potential mechanisms responsible for the development of cardiac dysfunction and heart failure. ${ }^{1}$ The major finding of this study suggests that cardiac high-energy phosphate metabolism, as measured by PCr:ATP ratio, is significantly correlated with measures of diastolic function and performance of the heart. In addition, the study findings demonstrate that cardiac metabolism, function and performance of the heart decline with age.

Results from previous studies evaluating changes in cardiac metabolism with age are conflicting. Okada et al and Köstler et $a l^{1819}$ reported significant decline in PCr and ATP levels in older subjects compared with younger subject. However, a decline in the PCr:ATP ratio was not observed. Our findings support recent studies reporting that $\mathrm{PCr}$ :ATP ratio decreases with age, ${ }^{11} 1720$ that is older women demonstrate significantly lower PCr:ATP ratio compared with younger women and that this decline in cardiac metabolism is negatively correlated with age. The decline in cardiac high-energy phosphate metabolism possibly reflects a number of age-associated changes including a decrease in mitochondrial oxidative phosphorylation, impaired myocardial lysosomes and decline in creatine kinase activity with age. ${ }^{21}$ Oxidative phosphorylation is responsible for $95 \%$ of cardiac ATP production; thus, a decline in oxidative phosphorylation will lead to the decrease in overall cardiac high-energy metabolism. Creatine kinase is a catalytic enzyme that is crucial in the regeneration of ATP

Table 4 Peak exercise gas exchange and central haemodynamic measures

\begin{tabular}{|c|c|c|c|c|}
\hline & All participants & Young ( $\leq 50$ years old, $n=20$ ) & Old ( $\geq 60$ years old, $n=15$ ) & $\mathrm{p}$ Value \\
\hline \multicolumn{5}{|l|}{ Peak exercise metabolic variables } \\
\hline Oxygen consumption(mL/min) & $1701.4 \pm 458.9$ & $1940.3 \pm 434.4$ & $1382.9 \pm 255.0$ & 0.00 \\
\hline Oxygen consumption (mL/kg/min) & $25.5 \pm 8.0$ & $28.6 \pm 8.8$ & $21.5 \pm 4.6$ & 0.00 \\
\hline Arterial-venous oxygen difference $\left(\mathrm{mLO}_{2}\right)$ & $12.5 \pm 2.9$ & $12.9 \pm 3.4$ & $11.9 \pm 2.2$ & 0.31 \\
\hline Respiratory exchange ratio & $1.2 \pm 0.1$ & $1.2 \pm 0.1$ & $1.2 \pm 0.1$ & 0.55 \\
\hline \multicolumn{5}{|l|}{ Peak exercise haemodynamics } \\
\hline Stroke volume index $\left(\mathrm{mL} /\right.$ beat $\left./ \mathrm{m}^{2}\right)$ & $51.8 \pm 9.9$ & $52.5 \pm 10.9$ & $50.8 \pm 8.7$ & 0.63 \\
\hline Cardiac output (L/min) & $13.9 \pm 3.2$ & $15.4 \pm 3.0$ & $11.9 \pm 2.3$ & 0.00 \\
\hline Cardiac index $\left(\mathrm{L} / \mathrm{min} / \mathrm{m}^{2}\right)$ & $8.0 \pm 1.8$ & $8.8 \pm 1.7$ & $7.1 \pm 1.3$ & 0.00 \\
\hline Systolic blood pressure (mm Hg) & $188.9 \pm 20.3$ & $182.5 \pm 21.1$ & $197.6 \pm 16.0$ & 0.03 \\
\hline Diastolic blood pressure $(\mathrm{mm} \mathrm{Hg})$ & $90.9 \pm 11.3$ & $90.5 \pm 12.7$ & $91.4 \pm 9.4$ & 0.81 \\
\hline Systemic vascular resistance $\left(\right.$ dyne $\left./\left(\mathrm{s} \cdot \mathrm{cm}^{5}\right)\right)$ & $750.9 \pm 191.8$ & $651.2 \pm 137.8$ & $883.8 \pm 174.6$ & 0.00 \\
\hline
\end{tabular}


from ADP and PCr, and its decline with age may lead to subsequent decrease in PCr level. ${ }^{18}$ Previous studies also reported an age-associated elevated increase in lipofuscine and decreased lysosomal efficiency leading to subsequent death of cardiac myocytes. ${ }^{17}$ Kates et al ${ }^{22}$ also reported a decline in myocardial fatty acid utilisation and oxidation with no change in glucose utilisation, which consequently lead to diminished overall cardiac high-energy phosphate metabolism.

Measures of cardiac function and performance change with age that is, decreased early-to-late diastolic filling ratio, cardiac output and cardiac power output, whereas systemic blood pressure and left-ventricular ejection fraction increased. These age-related haemodynamic changes have been previously reported. The increase in systolic blood pressure is due to accumulation of collagen and calcium in the large arteries, along with loss of elastic fibres from the medial layer. ${ }^{22} 23$ The thickening and stiffening of large arteries increase systemic vascular resistance, thus leading to raised systolic blood pressure. A decrease in early-to-late diastolic filling ratio with age occurs due to age-associated decline in left-ventricular early diastolic filling rate and increase in late diastolic ventricular filling. ${ }^{24}$ Early diastolic filling rate has been observed to decline progressively after the age of 20 and diminished to $50 \%$ by 80 years of age. ${ }^{24}$ An increase in late diastolic ventricular filling with age occurs due to increased atrial contribution to ventricular filling. ${ }^{25}$ Also, decline in diastolic function occurs due to a number of age-associated changes such as myocardial collagen accumulation, matrix proliferation, reduced calcium overload threshold and reduced ventricular length. ${ }^{26}$ These changes increase wall stress and reduce cardiac contractility, leading to subsequent decline in diastolic function.

This study shows that cardiac high-energy phosphate metabolism has a positive and significant relationship with early-to-late diastolic filling ratio, peak cardiac output, peak cardiac power output and cardiac reserve. This suggests that a decline in cardiac high-energy phosphate metabolism might lead to age-related decline in cardiac function and performance. It should also be noted that PCr:ATP ratio was negatively correlated with systolic blood pressure and systematic vascular resistance. As blood pressure is an important determinant of cardiac energy consumption, it potentially may
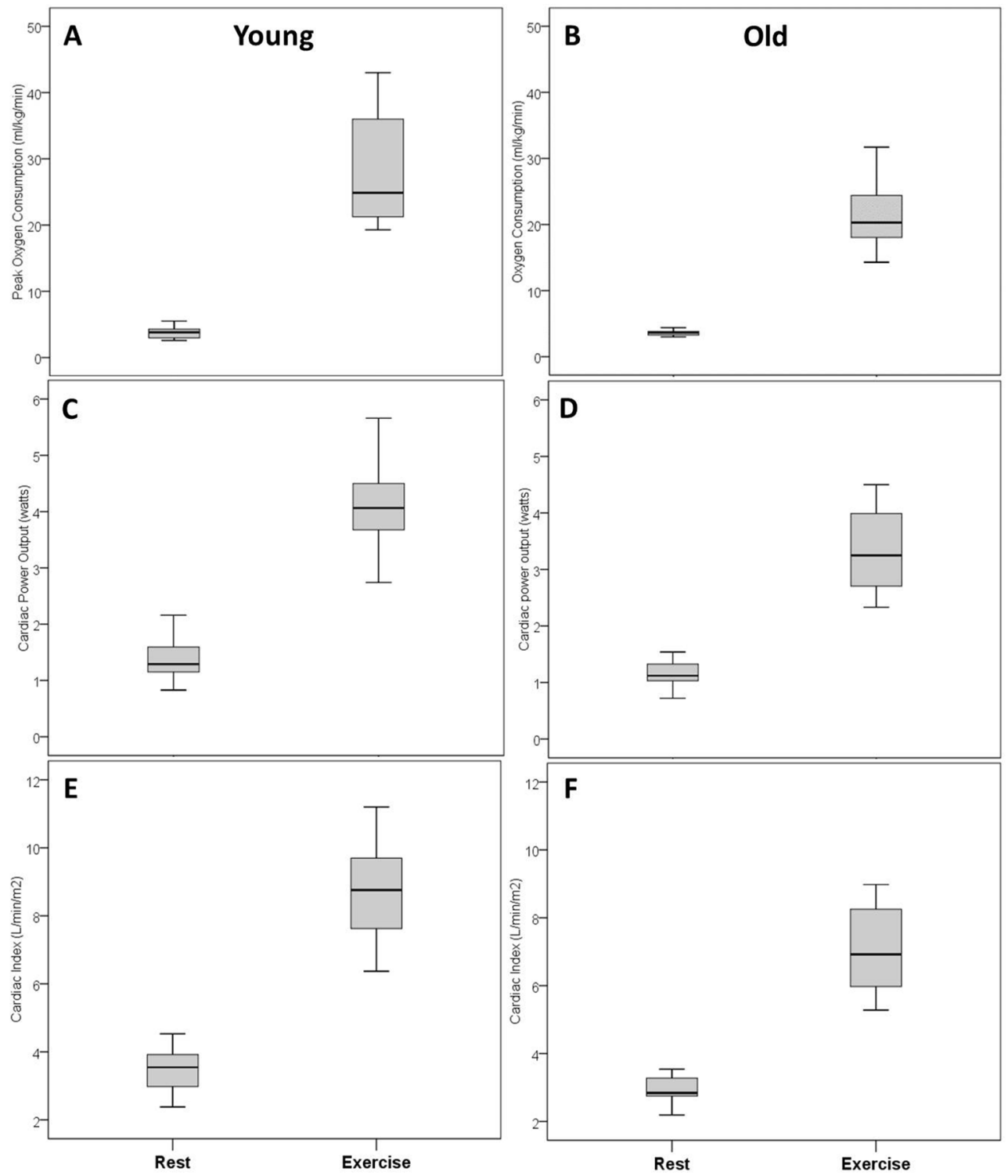

Figure 2 Cardiac and metabolic responses from rest to peak exercise in younger and older women: Oxygen consumption in young (A) and old (B); cardiac power output in young (C) and old (D); cardiac index in young (E) and Old (F). 

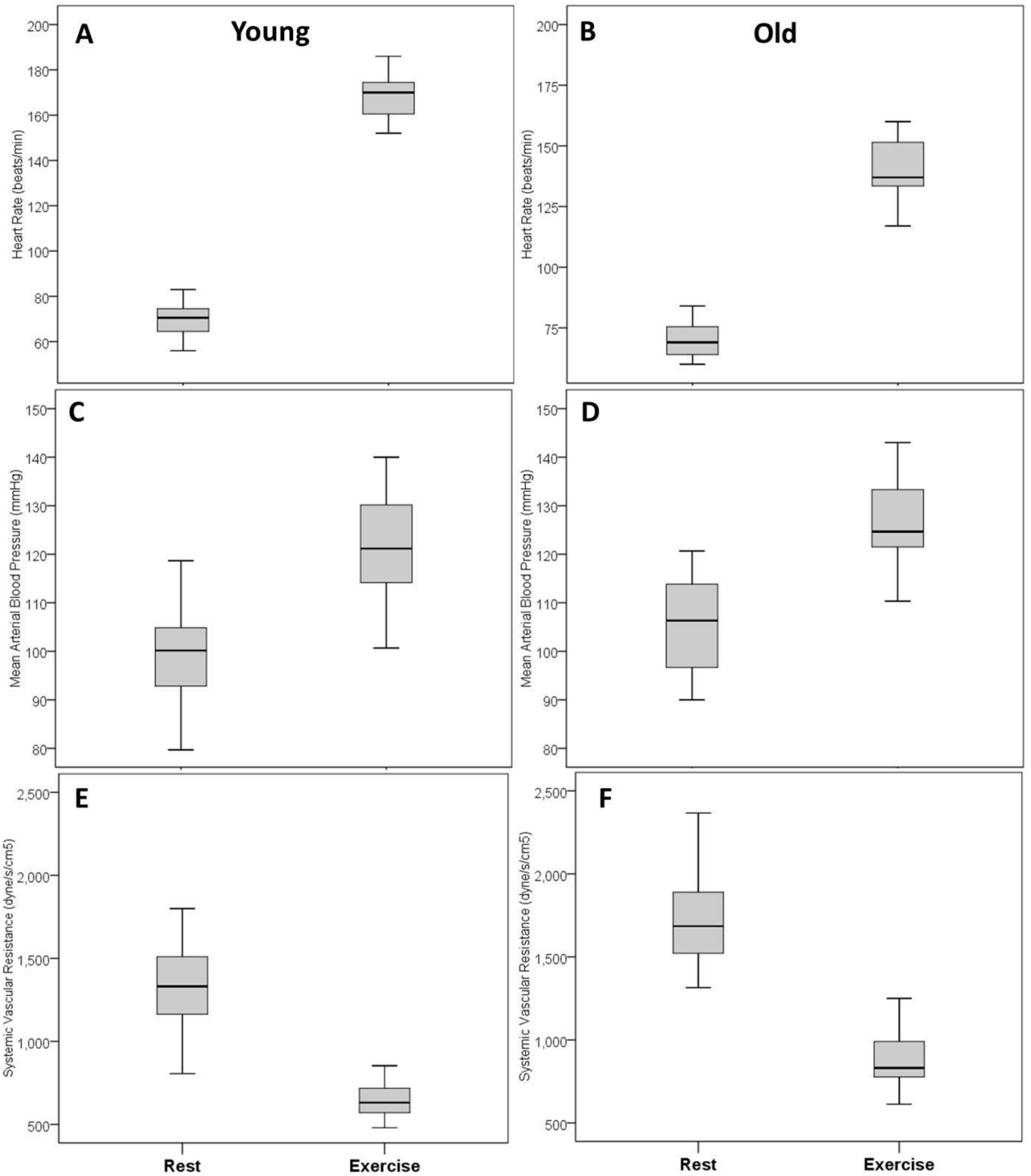

Figure 3 Cardiac responses from rest to peak exercise in younger and older women: Heart rate in young (A) and old (B); mean arterial blood pressure in young $(C)$ and old $(D)$; systematic vascular resistance in young $(E)$ and old $(F)$.

explain observed reduction in PCr:ATP ratio in older women, as previously suggested. ${ }^{27}$

This is the first human study to define the relationship between high-energy metabolism and overall maximal function and pumping capability of the heart represented by peak cardiac power. Peak cardiac power has been proposed to be the best indicator of overall cardiac function because it integrates both flow-generating and pressure-generating capacities of the heart, and as a such has been shown to be the strongest predictor of prognosis in heart failure. ${ }^{11}$

Our findings therefore support previous theory suggesting that diminished cardiac metabolism may play a significant role in mechanisms of development of cardiac dysfunction and heart failure. ${ }^{1}$ Previous animal studies have shown that alterations in cardiac high-energy phosphate metabolism lead to reduced contraction velocity, diminished systolic function and reduced cardiac contractility. ${ }^{67}$ However, the exact mechanism of how cardiac metabolism leads to diminished cardiac function and performance still need to be defined. Fundamentally, ATP hydrolysis is a key in driving the myosin head sliding movement along actin filament during contraction. ${ }^{28}$ Diminished cardiac high-energy phosphate metabolism will lead to reduced cardiac high-energy phosphates. The rates of myocardial ATP production must closely matched the cardiac metabolic demand. A halt in ATP production would deplete cardiac ATP stores in mere $15 \mathrm{~s}^{2}$ Therefore, decline in ATP production may affect cardiac contractility and viability and highly likely cause diminished cardiac function and performance. Moreover, a decline in $\mathrm{PCr}$ level might disrupt cardiac contractility. During inadequate oxygenation, $\mathrm{PCr}$ and ADP is rapidly converted into ATP through the rapid and reversible creatine kinase reaction. ${ }^{29}$ This is essential to maintain high levels of ATP and low levels of ADP. When energy demand outweighs supply, PCr level falls to maintain normal levels of ATP, with an increase in ADP level. ${ }^{529}$ Elevated free ADP inhibits intracellular enzymes and disrupts cardiac contractile function.

This study is not without limitations. Only women were studied due to the significant differences in age-associated changes in cardiac morphology and function between men and women as previously confirmed. ${ }^{9}$ Further larger mechanistic studies to include both women and men are required to provide better understanding of the interaction between cardiac energetics and function. 
A

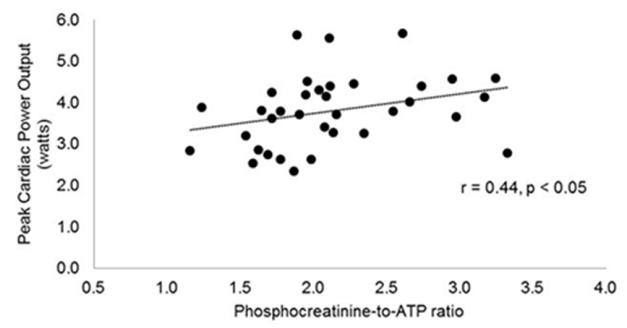

C

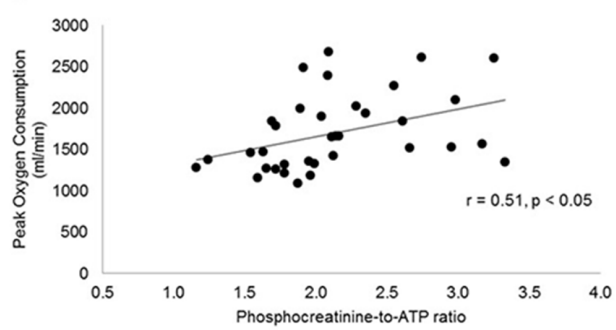

B

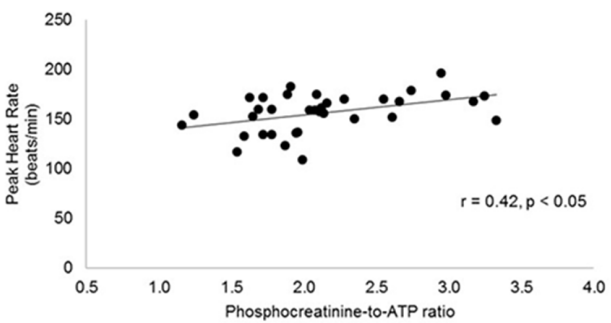

D

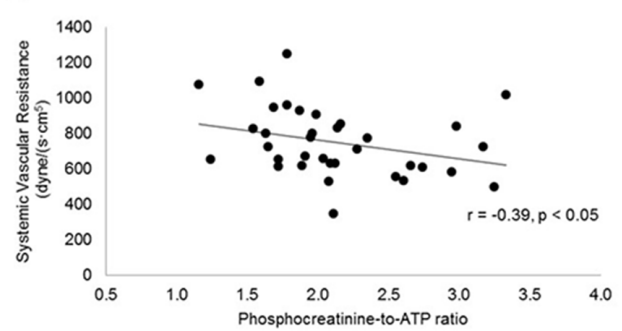

Figure 4 Relationship between cardiac high-energy phosphate metabolism and measures of cardiac function and performance obtained at peak exercise: Cardiac power output (A); heart rate (B); oxygen consumption (C) and systemic vascular resistance (D).

\section{CONCLUSION}

High-energy phosphate metabolism and performance of the heart decline with age. A significant positive relationship between PCr:ATP ratio and peak cardiac power output suggests that cardiac high-energy phosphate metabolism may be an important determinant of cardiac function and performance.

\section{Key messages}

\section{What is already known on this subject?}

Age-related decline in high-energy phosphate metabolism and cardiac performance may play an important role in the development of cardiac dysfunction and heart failure.

\section{What might this study add?}

This study demonstrates a significant positive relationship between phosphocreatine-to-ATP ratio and peak cardiac power, suggesting that cardiac high-energy phosphate metabolism may be an important determinant of cardiac function and performance.

\section{How might this impact on clinical practice?}

Therapeutic interventions known to improve cardiac metabolism may lead to improvement in overall cardiac function.

\author{
Author affiliations \\ ${ }^{1}$ Institute of Cellular Medicine, Newcastle Magnetic Resonance Centre, Medical \\ School, Newcastle University, Newcastle upon Tyne, UK \\ ${ }^{2}$ Cardiothoracic Department, James Cook University Hospital, Middleborough, UK \\ ${ }^{3}$ Faculty of Medicine, University of Novi Sad Novi Sad, Serbia and Institute of \\ Cardiovascular Diseases Vojvodina (Clinic for Cardiovascular Surgery), Sremska \\ Kamenica, Serbia \\ ${ }^{4}$ Department of Cardiology, Clinical Centre of Serbia, Medical School, University of \\ Belgrade, Belgrade, Serbia \\ ${ }^{5}$ Department of Cardiology, Freeman Hospital and Institute of Genetic Medicine, \\ Newcastle University, Newcastle upon Tyne, UK \\ ${ }^{6}$ Research Councils UK Centre for Ageing and Vitality, Newcastle University, UK \\ ${ }^{7}$ Wellcome Trust Centre for Mitochondrial Research, Institute of Neurosciences, \\ Newcastle University, Newcastle upon Tyne, UK \\ ${ }^{8}$ Clinical Research Facility, Royal Victoria Infirmary, Newcastle upon Tyne, UK
}

Correction notice This paper has been amended since it was published Online First. Owing to a scripting error, some of the publisher names in the references were replaced with 'BMJ Publishing Group'. This only affected the full text version, not the PDF. We have since corrected these errors and the correct publishers have been inserted into the references.

Acknowledgements We thank the participants for contributing to this study. In addition, we acknowledge the significant contribution from Mei Jun Chen, Research Nurse and Tim Hodgson, Carol Smith and Louise Morris, the research Radiographers.

Contributors DJ designed the study, collected and analysed data, and drafted the manuscript. MN and KGH contributed to data collection, analysis and interpretation, drafted the manuscript and approved the manuscript for submission. MIT, CE, MB, LV PMS, GAM and DMT contributed to the study design, critically revised and approved the manuscript for submission.

Funding This study was funded by the Newcastle National Institute for Health Research (NIHR) Biomedical Research Centre in Ageing and Chronic Conditions and Newcastle MRC Centre for Ageing and Vitality. KGH is supported by the UK Medical Research Council (G1100160), MIT by NIHR Senior Research Fellowship, LV by the Serbian Ministry of Education, Science and Technological Development (III41007 and ON174028) and EC HORIZON2020 SMARTool project (689068), and DGJ by Research Councils UK Centre for Ageing and Vitality (L016354).

Competing interests None declared.

Patient consent Obtained.

Ethics approval North East of England - Tyne and Wear South.

Provenance and peer review Not commissioned; externally peer reviewed.

Data sharing statement All data are included in this manuscript.

Open Access This is an Open Access article distributed in accordance with the terms of the Creative Commons Attribution (CC BY 4.0) license, which permits others to distribute, remix, adapt and build upon this work, for commercial use, provided the original work is properly cited. See: http://creativecommons.org/licenses/by/4.0/

(c) Article author(s) (or their employer(s) unless otherwise stated in the text of the article) 2018. All rights reserved. No commercial use is permitted unless otherwise expressly granted.

\section{REFERENCES}

1 Neubauer S. The failing heart--an engine out of fuel. N Engl J Med 2007;356:1140-51.

2 Weiss RG, Maslov M. Normal myocardial metabolism: fueling cardiac contraction. Advanced Studies Med 2004;4:S457-63.

3 Taegtmeyer H. Cardiac metabolism as a target for the treatment of heart failure. Circulation 2004;110:894-6. 
4 Beer M, Seyfarth T, Sandstede J, et al. Absolute concentrations of high-energy phosphate metabolites in normal, hypertrophied, and failing human myocardium measured noninvasively with (31)P-SLOOP magnetic resonance spectroscopy. J Am Coll Cardiol 2002;40:1267-74.

5 Ide T, Tsutsui H, Hayashidani S, et al. Mitochondrial DNA damage and dysfunction associated with oxidative stress in failing hearts after myocardial infarction. Circ Res 2001;88:529-35.

6 Hecker PA, Lionetti V, Ribeiro RF, et al. Glucose 6-phosphate dehydrogenase deficiency increases redox stress and moderately accelerates the development of heart failure. Circ Heart Fail 2013;6:118-26.

7 Kato T, Niizuma S, Inuzuka Y, et al. Analysis of metabolic remodeling in compensated left ventricular hypertrophy and heart failure. Circ Heart Fail 2010;3:420-30.

8 Williams SG, Cooke GA, Wright DJ, et al. Peak exercise cardiac power output; a direct Indicator of cardiac function strongly predictive of prognosis in chronic heart failure. Eur Heart J 2001;22:1496-503.

9 Goldspink DF, George KP, Chantler PD, et al. A study of Presbycardia, with gender differences favoring ageing women. Int J Cardiol 2009;137:236-45.

10 Jones DE, Hollingsworth K, Fattakhova G, et al. Impaired cardiovascular function in primary biliary cirrhosis. Am J Physiol Gastrointest Liver Physiol 2010;298:G764-73.

11 Jakovljevic DG, Papakonstantinou L, Blamire AM, et al. Effect of physical activity on age-related changes in cardiac function and performance in women. Circ Cardiovasc Imaging 2015;8:e002086.

12 Hollingsworth KG, Willis TA, Bates MG, et al. Subepicardial dysfunction leads to global left ventricular systolic impairment in patients with limb girdle muscular dystrophy 21. Eur J Heart Fail 2013;15:986-94.

13 Perkins RE, Hollingsworth KG, Eggett C, et al. Relationship between bioreactance and magnetic resonance imaging stroke volumes. Br J Anaesth 2016;117:134-6.

14 Jones TW, Houghton D, Cassidy S, et al. Bioreactance is a reliable method for estimating cardiac output at rest and during exercise. $\mathrm{Br} J$ Anaesth 2015;115:386-91.

15 Jakovljevic DG, Trenell MI, MacGowan GA. Bioimpedance and bioreactance methods for monitoring cardiac output. Best Pract Res Clin Anaesthesiol 2014;28:381-94.
16 Jakovljevic DG, Moore S, Hallsworth K, et al. Comparison of cardiac output determined by bioimpedance and bioreactance methods at rest and during exercise. $J$ Clin Monit Comput 2012;26:63-8.

17 Hollingsworth KG, Blamire AM, Keavney BD, et al. Left ventricular torsion, energetics, and diastolic function. Am J Physiol Heart Circ Physiol 2012;302:H885-92.

18 Okada M, Mitsunami K, Inubushi T, et al. Influence of aging or left ventricular hypertrophy on the human heart: contents of phosphorus metabolites measured by 31P MRS. Magn Reson Med 1998:39:772-82.

19 Köstler H, Landschütz W, Koeppe S, et al. Age and gender dependence of human cardiac phosphorus metabolites determined by SLOOP 31P MR spectroscopy. Magn Reson Med 2006;56:907-11.

20 Esterhammer R, Klug G, Wolf C, et al. Cardiac high-energy phosphate metabolism alters with age as studied in 196 healthy males with the help of 31-phosphorus 2-dimensional chemical shift imaging. PLoS One 2014;9:e97368.

21 Dutta D, Calvani R, Bernabei R, et al. Contribution of impaired mitochondrial autophagy to cardiac aging: mechanisms and therapeutic opportunities. Circ Res 2012;110:1125-38.

22 Kates AM, Herrero P, Dence $C$, et al. Impact of aging on substrate metabolism by the human heart. J Am Coll Cardiol 2003;41:293-9.

23 Challah M, Nadaud S, Philippe M, et al. Circulating and cellular markers of endothelia dysfunction with aging in rats. Am J Physiol 1997;273:H1941-8.

24 Gerstenblith G, Frederiksen J, Yin FC, et al. Echocardiographic assessment of a normal adult aging population. Circulation 1977;56:273-8.

25 Miyatake K, Okamoto M, Kinoshita N, et al. Augmentation of atrial contribution to left ventricular inflow with aging as assessed by intracardiac doppler flowmetry. Am J Cardiol 1984;53:586-9.

26 Lakatta EG, Levy D. Arterial and cardiac aging: major shareholders in cardiovascular disease enterprises: part II: the aging heart in health: links to heart disease. Circulation 2003;107:346-54.

27 Fragasso G, De Cobelli F, Spoladore R, et al. Resting cardiac energy metabolism is inversely associated with heart rate in healthy young adult men. Am Heart $J$ 2011;162:136-41.

28 Sherwood L. Human Physiology: from cells to Systems. 7th ed. United States: Brooks/ Cole, 2010.

29 Ingwall JS, Kramer MF, Fifer MA, et al. The creatine kinase system in normal and diseased human myocardium. N Engl J Med 1985;313:1050-4. 